\begin{tabular}{|c|c|}
\hline Research & 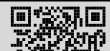 \\
\hline Article & 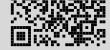 \\
\hline
\end{tabular}

\title{
Deep Learning-Based Hybrid Fuzz Testing
}

\author{
Fengjuan Gao (高凤娟) $)^{1,2}$, Yu Wang (王豫) $)^{1,2}$, Lingyun Situ (司徒凌云) ${ }^{3}$, \\ Linzhang Wang (王林章) ${ }^{1,2}$ \\ 1 (State Key Laboratory for Novel Software Technology at Nanjing University, Nanjing 210023, China) \\ 2 (Department of Computer Science and Technology, Nanjing University, Nanjing 210023, China) \\ 3 (School of Information Management, Nanjing University, Nanjing 210023, China) \\ Corresponding author: Linzhang Wang, lzwang@nju.edu.cn
}

\begin{abstract}
With the rapid development of software techniques, domain-driven software raises new challenges in software security and robustness. Symbolic execution and fuzzing have been rapidly developed in recent decades, demonstrating their ability in detecting software bugs. Enormous detected and fixed bugs prove the feasibility of the two methods. However, it is still a challenging task to combine the two methods due to their respective weaknesses. State-ofthe-art techniques focus on incorporating the two methods such as using symbolic execution to solve paths when fuzzing gets stuck in complex paths. Unfortunately, such methods are inefficient because they have to switch to fuzzing (resp. symbolic execution) when performing symbolic execution (resp. fuzzing). This paper presents a novel deep learning-based hybrid testing method using symbolic execution and fuzzing. The method tries to predict paths that are suitable for fuzzing (resp. symbolic execution) and use the fuzzing (resp. symbolic execution) to reach the paths. To further enhance effectiveness, this paper also proposes a hybrid mechanism to make them interact with each other. The proposed approach is evaluated on the programs in LAVA-M, and the results are compared with those in the case of using symbolic execution or fuzzing independently. It achieves more than $20 \%$ increase in branch coverage and 1 to 13 times increase in the path number and uncovers 929 more bugs.
\end{abstract}

Keywords software testing; deep learning; path representation; symbolic execution; fuzzing; hybrid testing

Citation Gao FJ, Wang Y, Situ LY, Wang LZ. Deep learning-based hybrid fuzz testing, International Journal of Software and Informatics, 2021, 11(3): 335-355. http://www.ijsi.org/1673-7288/261.htm

Software is the driver for the development of a new generation of information technology. With the advances in Internet of Things (IoT), cloud computing and Artificial Intelligence (AI), software that is geared to the needs of these domains keeps making progress. However, problems regarding software quality and security emerge in the course. Domain-driven software faces the challenge of increase in software amount. More importantly, the intrinsic features of applied domains, such as dynamic organization unit, ubiquitous access to networks, and digital twins common in industrial automation control and manufacturing, impose higher demands on

This is the English version of the Chinese article “基于深度学习的混合模糊测试方法. 软件学报, 2021, 32(4): 988-1005. doi: 10.13328/j.cnki.jos.006225”.

Funding items: National Natural Science Foundation of China (62032010); Postgraduate Research \& Practice Innovation Program of Jiangsu Province

Received 2020-09-13; Revised 2020-10-26; Accepted 2020-12-19; IJSI published online 2021-09-25 
production efficiency and security. Defects of software, its own inherent problem, remain a threat to software robustness and security.

Software defects emerge in the process of programming, which may be brought in by improper development or inexperience of the programmers. Software with defects may cause unexpected effects. In serious cases, enterprises may suffer from massive economic loss, life and property security of people may even be in danger. Hence, earlier uncovering of software defects is more beneficial for lowering the cost of defect repairing.

Fuzzing and symbolic execution are two main stream and effective methods for detecting software defects. Fuzzing is effective to find software defects by constantly feeding unexpected data as inputs to the tested program and monitoring whether the program shows abnormal behaviors. It is a kind of automated software testing technique based on faulty inputs. It feeds a large number of auto-generated test cases to the program to find potential security defects, such as buffer overflow. Symbolic execution is a program analysis technique which is able to obtain the inputs that execute the designated paths by analyzing the program. In the case of symbolic execution to analyze a program, the program will use symbolic values as inputs rather than concrete values that are common for general programs. When reaching the object code, the analyzer can obtain the corresponding path constraints. Then, the constraint solver helps determine the concrete inputs that can trigger the object code. Fuzzing and symbolic execution have their respective limitations. Since fuzzing cannot recognize path constraints, in case constraints are narrow (e.g., whether two strings are equal), it cannot effectively cover the path $^{[1]}$. In comparison, although symbolic execution can cope with narrow constraints, it cannot fully and rapidly cover the program space ${ }^{[2]}$ (such as a deep portion of the program) because of path explosion and constraint solving.

So far, there have been some methods integrating symbolic execution and fuzzing ${ }^{[1-7]}$. Once one method gets stuck, it will switch to another method. For example, Driller ${ }^{[1]}$ turned to symbolic execution in case fuzzing got stuck when encountering special boundary conditions (such as narrow paths meeting specific inputs) and then fed the solved results back to fuzzing. However, there remains a common problem, namely that another method will not be used unless the preferred method becomes ineffective, which will lower the overall efficiency.

To tackle this problem, we propose a deep learning-based hybrid testing method integrating symbolic execution and fuzzing, called SmartFuSE (Smart Fuzzing and Symbolic Execution). Given a program, we construct the graph representation of the paths of the program. A neural network model is employed then to predict whether a path is suitable for fuzzing or symbolic execution. For the path set suitable for symbolic execution (resp. fuzzing), symbolic execution (resp. fuzzing) will be used to prioritize the execution of such a path set. Moreover, we propose a hybrid method to make symbolic execution and fuzzing interact with each other, thus further increasing the overall coverage. We carry out tests on four programs in LAVA-M. The results show that, compared with fuzzing or symbolic execution alone, our hybrid method improves the branch coverage by more than $20 \%$, increases the number of paths by 1 to 13 times, and uncovers 929 more defects.

This paper offers the following contributions.

- It proposes path prediction methods with deep learning-based symbolic execution and fuzzing.

- It introduces guided symbolic execution and guided fuzzing. Guided symbolic execution (resp. fuzzing) has the priority to explore the path set suitable for symbolic execution (resp. fuzzing) which is predicted by the model.

- It proposes a hybrid testing method integrating symbolic execution and fuzzing.

- It develops the prototype tool SmartFuSE, the effectiveness of which is confirmed by 
experiments.

This paper is structured as follows. To begin with, we introduce research background, including symbolic execution, fuzzing, and Graph Neural Networks (GNNs). Then, we outline the framework of SmartFuSE. In addition, we describe how to represent and collect data and train the GNN model. Subsequently, we illustrate how to guide the hybrid testing method integrating symbolic execution and fuzzing with the GNN model. Further, we describe the implementation of SmartFuSE and evaluate its effectiveness and performance with experiments. Then we expound on relevant studies. At last, conclusions are drawn from this work.

\section{Preliminary}

\subsection{Symbolic execution}

Symbolic execution, a common software analysis technique, was first proposed by King et al. in 1975 or so $^{[8]}$. KLEE $^{[9]}$ is one of the most extensively applied symbolic execution engines. The key idea of symbolic execution lies in that symbolic values serve as inputs instead of concrete values and a symbolic expression is used to represent the value of the program variable correlated with a symbolic value. In the presence of a branch instruction, the program executor will correspondingly search each branch. The branch condition is then added to $\pi$, which is the set of program statuses saved by symbolic execution, in order to represent the constraints of the current path. After collecting the path constraint $\pi$, a constraint solver is employed to solve whether the constraint can be satisfied to check the reachability of the path. If the path constraint can be satisfied, it proves that the path can be reached and therefore should be further explored. Otherwise, it proves that the path cannot be reached and should not be further explored.

In Listing 1, we symbolically execute the program by making the parameters of the main function symbolic. When the execution reaches the if statement in the fourth line, symbolic execution will fork a status for each of the two branches. Then, the path constraint $x^{*} l$ en $==30$ is added to the status of the true branch, and the constraint $x^{*} l$ en $\quad !=30$ is added to the status of the false branch. As the execution path becomes deeper, the path constraints in the symbolic execution will be increasingly complicated, and constraint solving will be more time-consuming. Especially, executing the loop statement in the 12th line results in a state explosion. Probably, symbolic executors cannot well explore the space of other paths further.

Listing 1 Example program

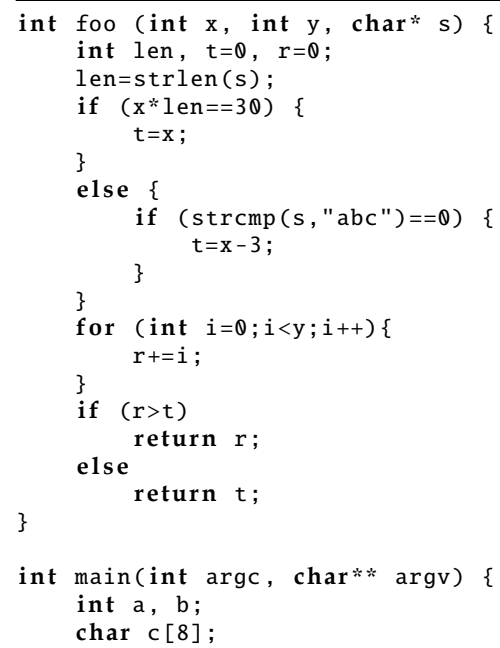


FILE *fp=fopen (argv[1], "r"); // read mode

fscanf (fp, "\%d \%d \%s", \&a, \&b, c) ;

fclose ( $f p)$;

\}

return foo $(a, b, c)$;

\subsection{Fuzzing}

Fuzzing, a common software test technique, was first proposed by Millor et al. in $1988^{[10,11]}$. The core idea of fuzzing is feeding random data automatically or semi-automatically generated as input into a program, and detecting defects by monitoring the abnormal behaviors, such as crashes and assertion fails, of the execution of the program. In general, fuzzing is applied to test the security vulnerabilities in software or computer systems. As an automated or semi-automated process, fuzzing includes repeatedly monitoring target software and providing mutated data to it. The method of mutating test cases is critical to the fuzzing process. The tool for generating mutated data is called fuzzer.

American Fuzzy Lop (AFL) ${ }^{[12]}$ is a coverage-guided fuzzing tool developed by the security researcher Michael Zalewski. It is one of the most extensively applied fuzzing tools. Through code instrumentation in programming, AFL logs code coverage. Then, the test case in the input queue is mutated in line with specific strategies, such as bit flipping, byte copying, and byte deletion. If the mutated input can increase code coverage, such an input is reserved in the input queue. The above process keeps repeating until the executor triggers a crash. Corresponding test cases will be reported and logged.

For the example in Listing 1, through fuzzing, AFL can generate, within seconds, the input that can cover the code in the fifth line, i.e., the true branch of if $\left(x^{*} l e n==30\right)$. However, AFL cannot generate, even within $10 \mathrm{~h}$, the test input that can cover the code in the tenth line, i.e., the true branch of if $(\operatorname{strcmp}(s, " a b c ")==0)$.

\subsection{GNN}

In this paper, we employ a mutation of GNNs, that is, Gated Graph Neural Networks (GGNNs). As a model based on GNNs, GGNNs adopts a gated mechanism similar to Gated Recurrent Unit (GRU, a mutation of Long-Short Term Memory (LSTM), which can solve longdistance dependence) to expand the architecture. In this way, the network can be learned through Back Propagation (BP).

We first introduce GNNs. In the graph $G=(V, E, M), V$ denotes the set of nodes, $E$ the set of edges, and $M$ the set of vectors, i.e., $\left(\mu_{v 1}, \cdots, \mu_{v m}\right)$, in which $\mu_{v 1}$ is the real number used for logging the representation of the node $v$ in the graph. GNN updates the representation of each node in line with the following propagation rule.

$$
\mu_{v}^{(l+1)}=h\left(\left\{\mu_{u}^{(l)}\right\} u \in N^{k}(v), k \in\{1,2, \cdots, K\}\right)
$$

Specifically, the new representation of the node $v$ is obtained through the integrated calculation of the representations of neighbor nodes (in the form of vectors). $N^{k}(v)$ denotes all the $v$-connected neighbor nodes whose edge type is $k$, i.e., $N^{k}(v)=\{u \mid(u, v, k) \in E\}$ $\cup\{u \mid(v, u, k) \in \varepsilon\}$. Then, $\forall v \in V$, the procedure is repeated for $L$ times to upgrade $u_{v}$ as $u_{v}^{(L)}$. Most GNNs calculate the independent representations of different types of edges and then merge these representations to obtain the final node representation ${ }^{[13]}$.

$$
u_{v}^{(l+1), k}=\sigma_{1}\left(\sum_{u \in N^{k}(v)} W_{2} \mu_{u}^{(l)}\right), \forall k \in\{1,2, \cdots, K\}
$$




$$
\begin{aligned}
u_{v}^{(l+1)} & =\sigma_{2}\left(W_{3}\left[\mu_{u}^{(l), 1}, \mu_{u}^{(l), 2}, \cdots, \mu_{u}^{(l), K}\right]\right) \\
u_{v}^{(0)} & =W_{1} u_{v}
\end{aligned}
$$

Formula (4) shows the initial situation, i.e., $l=0$ and $\mu_{v}$ denotes the initial vector. Matrices $W_{1}, W_{2}$, and $W_{3}$ are the variables to be learned. $\sigma_{1}$ and $\sigma_{2}$ are non-linear activation functions. To further improve the capability of the model, Li et al.$^{[14]}$ propose GGNNs. The difference between a GNN and a GGNN is that the latter uses GRN ${ }^{[15]}$ to instantiate $h$ in Formula (1). The following two formulas explain how a GGNN is updated.

$$
\begin{aligned}
\tilde{m}_{v}^{l} & =\sum_{u \in N(v)} f\left(\mu_{u}^{(l)}\right) \\
\mu_{v}^{(l+1)} & =\operatorname{GRU}\left(\tilde{m}_{v}^{l}, \mu_{u}^{(l)}\right)
\end{aligned}
$$

To update the representation of node $v$, we use the $f(\cdot)$ function to represent all the neighbor nodes $N(v)$ of the node $v$ and then sum them up to produce $\tilde{m}_{v}^{l}$ (Formula (5)). Afterward, with $\tilde{m}_{v}^{l}$ and the representation $\mu_{v}^{(l)}$ of the present node $v$, GRU calculates the next new representation $\mu_{v}^{(l+1)}$ (Formula (6)). Similarly, such a propagation will be repeated for a certain number of times. The last obtained representation of the node is used as its final representation.

\section{SmartFuSE Framework}

The SmartFuSE framework is shown in Figure 1. It mainly consists of two modules, i.e., model training and hybrid testing. For the model training module, we first collect certain samples, which constitute path sets and the time for symbolic execution and fuzzing to reach each path (if they cannot reach the path, the time is recorded as infinite). With the collected information of samples, SmartFuSE encodes the paths and represent them through a graph. At last, we train the GNN model with the samples. The output of the module is the model that has been trained.

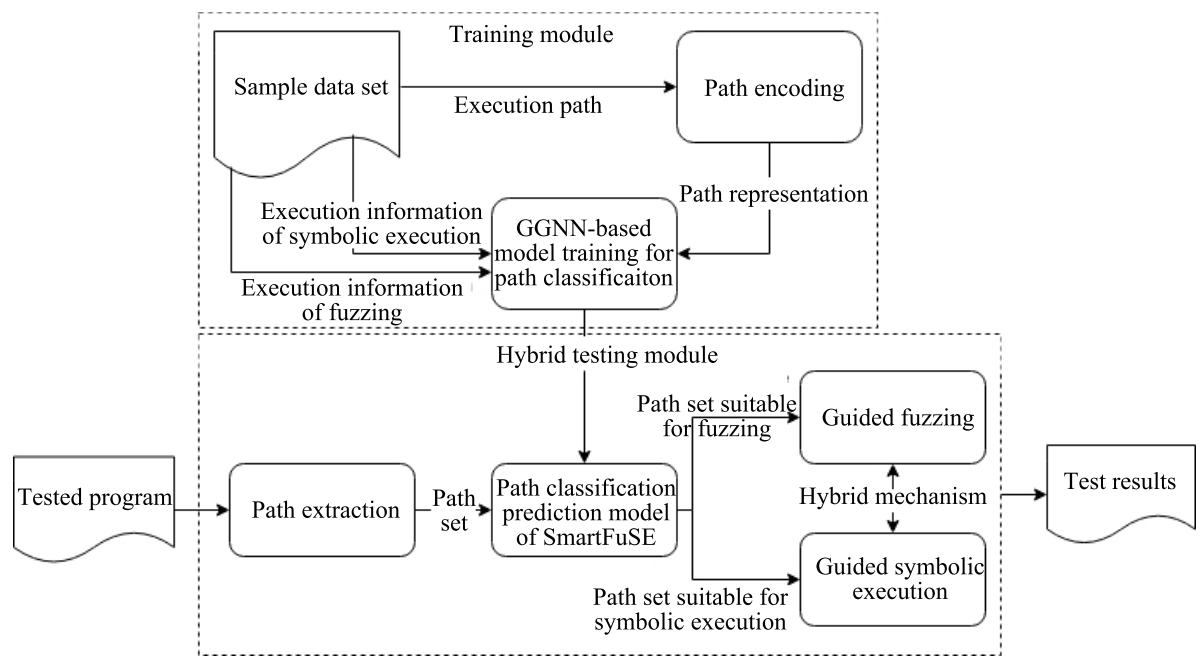

Figure 1 Overall framework of our method

The input of the hybrid testing module is a tested program. For the tested program, as the number of paths is large, we first extract some paths with certain depths and then choose 
some deeper paths so as to control the number of paths to be predicted. Afterward, for the analyzed paths, with the model obtained from the model training module, SmartFuSE predicts the suitability of each path for fuzzing or symbolic execution. For the paths suitable for fuzzing, SmartFuSE will use fuzzing to execute these paths. For the paths suitable for symbolic execution, SmartFuSE will exploit symbolic execution to prioritize these paths.

Incorrect prediction may cause some paths suitable for fuzzing (resp. symbolic execution) to be switched to symbolic execution (resp. fuzzing). Thus, SmartFuSE aggregates the paths suitable for fuzzing (resp. symbolic execution) and switch those paths that cannot be covered by fuzzing (resp. symbolic execution) to symbolic execution (resp. fuzzing) for the performance improvement. This is known as path switch, which can further enhance path coverage.

\section{Model Training}

In the following, we will introduce the data set collection, path encoding, and GGNN in succession.

\subsection{Data set}

In machine learning, the training data set plays a crucial role. How to effectively generate a data set remains a challenge. In general, people adopt existing data sets, which can provide basic data and facilitate the evaluation of experimental effects. However, there are no data sets designated for symbolic execution or fuzzing.

To collect data for training a model, we need to collect and analyze data from existing projects. The collected data is expressed as a triplet $\langle$ Path, SE_Time, Fuzz_Time $\rangle$, in which Path $=\left(l_{1}, l_{2}, \cdots, l_{n}\right)$ denotes one path of the program; $l_{i}(i \in\{1, \cdots, n\})$ denotes the Line Of Code (LOC) that the path covers, and information includes the file name and specific LOC number; SE_Time refers to the time taken by symbolic execution to cover Path for the first time, and similarly, Fuzz_Time denotes the time required by fuzzing to cover Path for the first time. If symbolic execution or fuzzing does not cover the path within the designated time, then the corresponding covering time is treated as positively infinite. In the absence of looping, one Path can be not correlated with any other Path. In the presence of looping, however, multiple Paths will be generated. These Paths are correlated with each other, with their only difference being the number of looping. In this context, the data quantity of data sets increases by a large margin, which thus affects the proportion of other data. To solve this problem, we limit the number of correlated Paths through a configuration item, with the default value being 3 .

\subsection{Representation of program execution paths}

Depending on the Abstract Syntax Tree (AST), the program paths are represented on control flow and data flow graphs. The reached paths are colored so that they can be distinguished from uncovered paths. $G=\langle V, E\rangle$ denotes the representation graph of one Path in the program. Each node in $V$ corresponds to an AST node. Node types comprise FunctionDecl, UnaryOperator, BinaryOperator, IfStmt, ForStmt, and WhileStm. Each edge in $E$ denotes the relationship between nodes. Edge types are composed of AST edge, control flow edge, data dependence edge, and path identification edge. The control flow edge refers to the edge in the Control Flow Graph (CFG). For the representation of the control flow edge, SmartFuSE extracts the function calling in the path in accordance with execution paths. Then, the $\mathrm{CFG}$ of each function is analyzed in line with such calling relationship. These CFGs are connected according to the function calling relationship, and an integral CFG can be obtained, which is referred to as Interprocedural Control Flow Graph (ICFG). Afterward, SmartFuSE further analyzes data dependences on the ICFG. For each variable on the ICFG, SmartFuSE adds a data dependence edge of the variable. In other words, such a dependence is represented by an additional edge on 
the ICFG. In addition, a path identification edge is introduced to show which statements have been executed by such a path. If the path identification edge and the control flow edge co-exist, only the path identification edge is reserved to indicate coloring information of the execution path.

Algorithm 1 shows the building process of the program path graph. First, on the basis of AST, ICFG (Line 1) is built, and then data dependence ${ }^{[16]}$ is analyzed on the ICFG. The data dependence edge is added to form a new graph DF-ICFG (Line 2). Later, except for $l_{1}$ in the Path, the corresponding control flow node $b b$ is extracted for each line number $l$ in Path, and the control flow node of its previous line prevLine is prevbb. The path identification edge from $p r e v b b$ to $b b$ is added to DF-ICFG (Line 4-Line 12). At last, through trimGraph, only CFG containing the path identification edge is reserved, otherwise, CFG is deleted from the corresponding ICFG. In the end, we obtain the graph representation PathGraph of the program Path (Line 13).

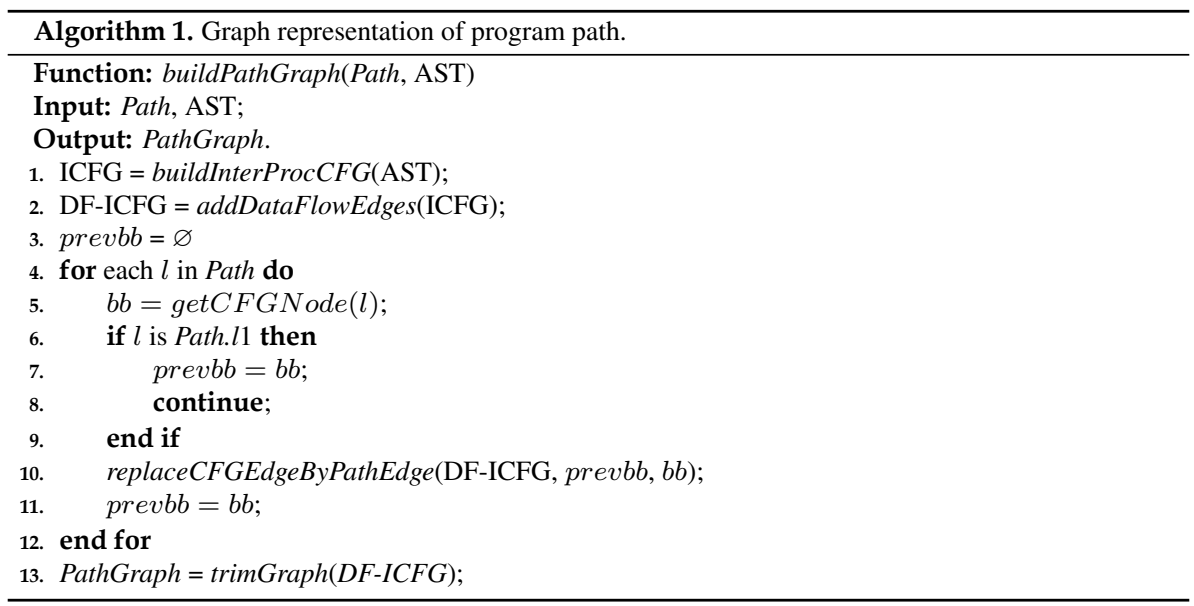

Figure 2 shows the path representation of an execution path (covering code behaviors $\langle 4,8,9,12,15,16\rangle)$ of the function |fool in the example program in Listing 1 . The node of the execution path corresponds to an AST node. Each edge without an arrow is an edge in AST; each black solid arrow denotes a control flow edge; each red solid arrow with hollow arrowhead denotes a path identification edge; each green dotted arrow denotes a data dependence edge.

\section{Hybrid Testing}

The hybrid testing module consists of path extraction and classification, guided symbolic execution, and guided fuzzing.

\subsection{Path extraction and classification}

The number of program paths is usually tremendous. Thus, SmartFuSE extracts only some paths for a high efficiency. As Algorithm 2 shows, in line with the given path depth (depth), SmartFuSE employs the extractPathsByBranchCov strategy to traverse all the paths reaching such a depth (Line 6-Line 7). The extractPathsByBranchCov function is responsible for extracting the path set able to cover statements by choosing statements randomly. However, the information of the deeper part of a path has important reference value as well. Hence, for each path reaching the designated depth, SmartFuSE analyzes its deeper part. This lowers the requirement of path extraction and thus reduces the number of paths to be extracted. 


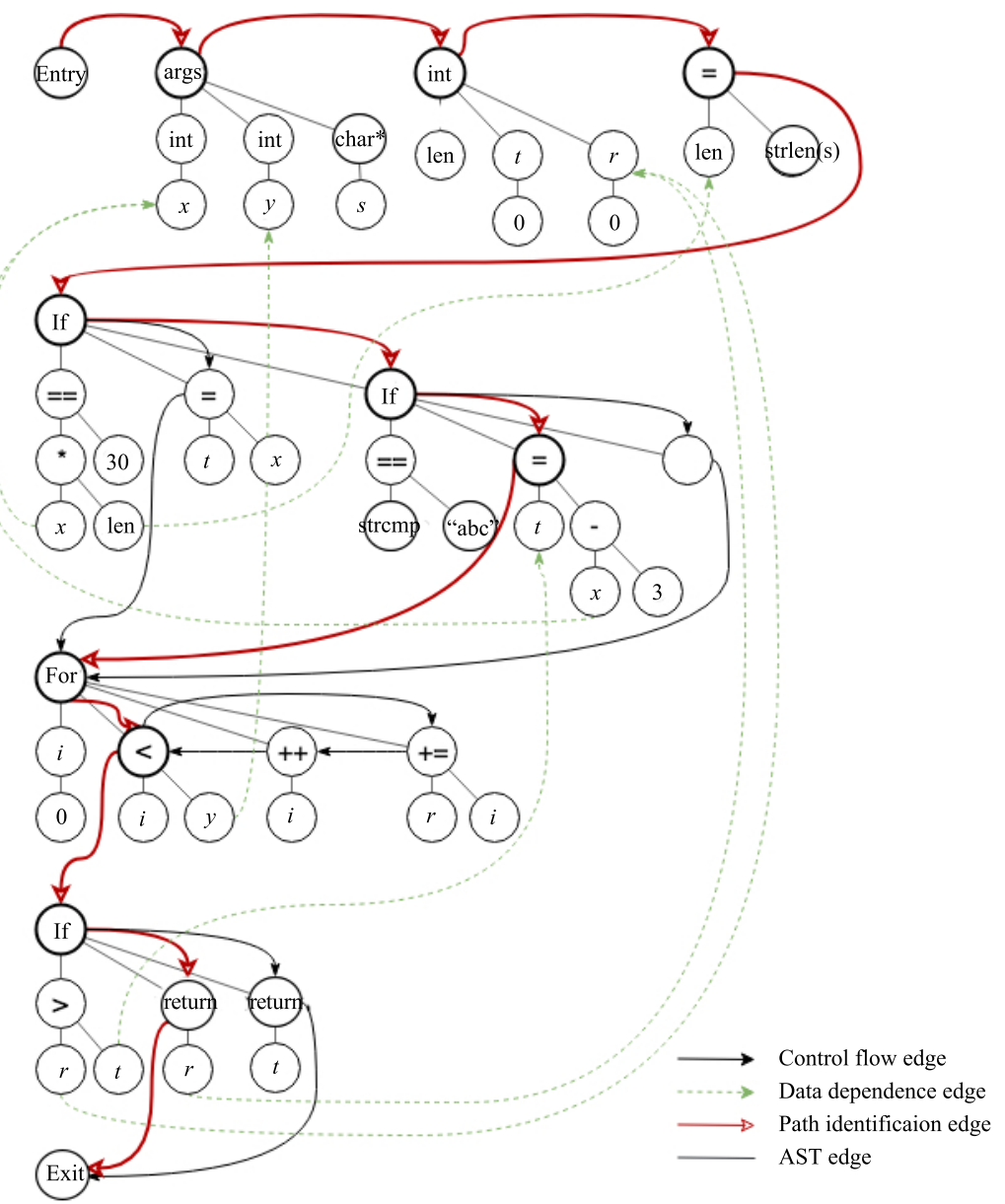

Figure 2 Example of path representation

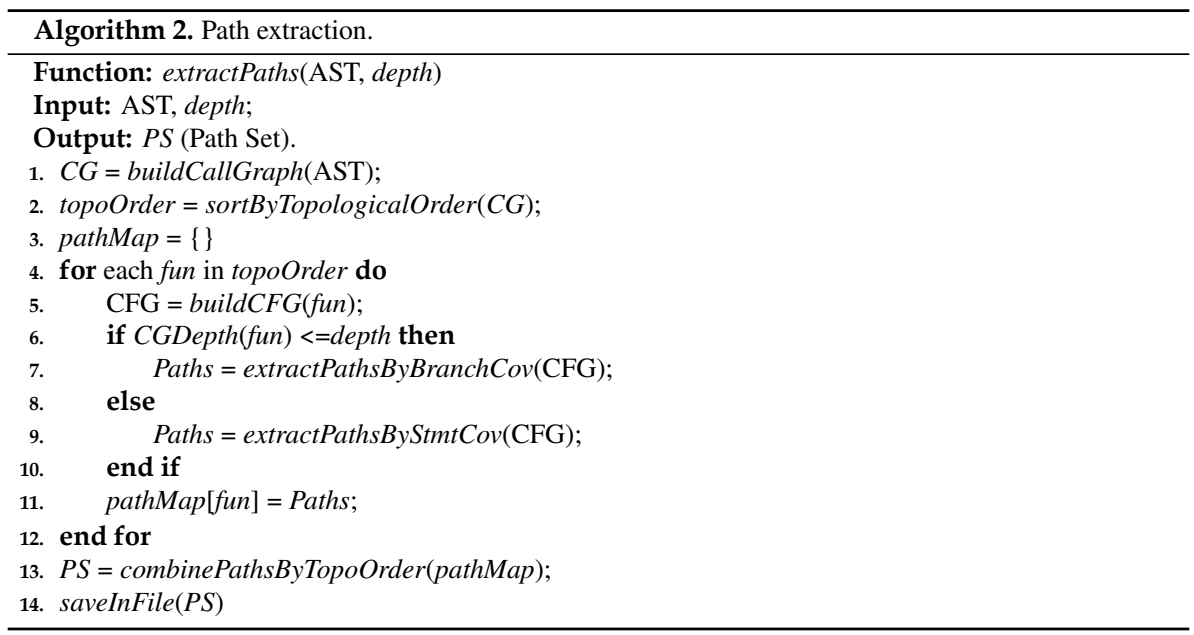


In this paper, the extractPathsByStmtCov strategy is adopted, namely that the path set that can realize branch coverage is extracted through random branch selection (Line 9).

A path set is obtained through path extraction. Then, according to Algorithm 1 mentioned above, we build the graph representation of each path in the set. SmartFuSE uses the trained model to predict the graphs of the extracted paths. The score of the predicted paths is represented as a pair $(X, Y)$, where $X$ stands for the score of paths suitable for fuzzing, while $Y$ represents the score of paths suitable for symbolic execution, with $X+Y=1$. We categorize the results of the path classification into three types, i.e., paths suitable for fuzzing $(X>Y)$, paths suitable for symbolic execution $(X<Y)$, and paths that are not certainly suitable for fuzzing or symbolic execution $(X \approx Y)$. In terms of the third type, the difference in values between $X$ and $Y$ is rather small (for example, less than 0.05). If a path is suitable for one method, the information of the path is transferred to that method. In case of uncertainty, it would be transferred to both methods. The paths suitable for symbolic execution constitute the SEPathSet set, and those suitable for fuzzing make up the FuzzPathSet set.

\subsection{Guided symbolic execution}

After the SEPathSet is predicted, SmartFuSE guides the symbolic execution to prioritize the path set. On the basis of conventional symbolic execution, the path set is added as input. A higher priority is given to the executive state correlated with these target paths so that the target paths can be explored first.

As Algorithm 3 shows, in guided symbolic execution, a SEPathSet set suitable for symbolic execution predicted by path extraction and classification serves as input. At each moment of the symbolic execution, the most prioritized state is selected from the executive state pool for execution. If the priority of all states in the executive state pool is the same, state selection (GuidedSymbolicExecution Line 6) is carried out according to the searching strategies (such as DFS, BFS, random path, and random state) adopted by the symbolic execution engines. When the execution reaches the branch statement (ExecuteInstruction Line 1), the state is copied and assigned to two branches (ExecuteInstruction Line 2-Line 5), and the priorities of the two states are updated (ExecuteInstruction Line 6-Line 7). In other words, for each state, we first judge whether the first statement of its basic block is in SEPathSet. If the branch is in SEPPathSet, we update the priority of its corresponding executive state (UpdatePrioriy Line 1, Line 2).

\subsection{Guided fuzzing}

In the same vein, after FuzzPathSet is predicted, SmartFuSE guides fuzzing to first explore the set. The paths of FuzzPathSet that cross more nodes have higher weight. By calculating the overall weight of nodes which the paths cross in the newly generated test case, we find that as the overall weight increases, the priority is enhanced. At each moment of fuzzing, the test case with the highest priority is selected for the next round of fuzzing.

Algorithm 4 shows the guiding process. In guided fuzzing, FuzzPathSet consisting of target paths serves as the input. First, SmartFuSE computes the weight of each node (GuidedFuzzing Line 1). The weight of each node is initialized to 0 . Then, for each node $V$, if the number of paths in FuzzPathSet which cross the node is $N$, then the weight of the node in $V$ is $N$ ( initWeight Line 1-Line 8). Next, we compute the priority of each seed in the test input list Seeds (GuidedFuzzing Line 3-Line 4). At first, we obtain the priority of the seed computed by the original fuzzer, which is then normalized to the interval $[0,1]$. The normalized value $(v)$ is taken as the initial priority of the seed in FuzzPathSet (computePriority Line 1). On the basis of $v$, we take the sum of the weights of the nodes in paths that the seed passes as the final priority of the seed (computePriority Line 2-Line 5). In this way, fuzzing is guided to 
first explore the paths in FuzzPathSet. After the paths of FuzzPathSet are explored, seeds are selected in line with the priority computed by the original fuzzer for subsequent fuzzing. After the priority of each seed in the test input list Seeds is computed, the seed with the highest priority, selectedSeed (GuidedFuzzing Line 6), is subjected to mutation to yield the new test input newSeed (GuidedFuzzing Line 7), and selectedSeed is removed from Seeds (GuidedFuzzing Line 8). Then, the new test input newSeed is executed. If newSeed can cover some new code branch, it is added to the test input list Seeds (GuidedFuzzing Line 9-Line 11). Meanwhile, if the path of the newSeed is in the FuzzPathSet, the weight of all nodes in the path is reduced by 1 (GuidedFuzzing Line 14-Line 15). In this way, fuzzing will no longer be guided to explore the paths covered by FuzzPathSet. When the weight of all nodes is reduced to 0, it implies that all paths of FuzzPathSet have been covered. Then, according to the algorithm in computePriority, the priorities computed by the original fuzzer are utilized to select seeds for subsequent fuzzing, i.e., to try to cover new codes and branches that have not been covered by SmartFuSE.

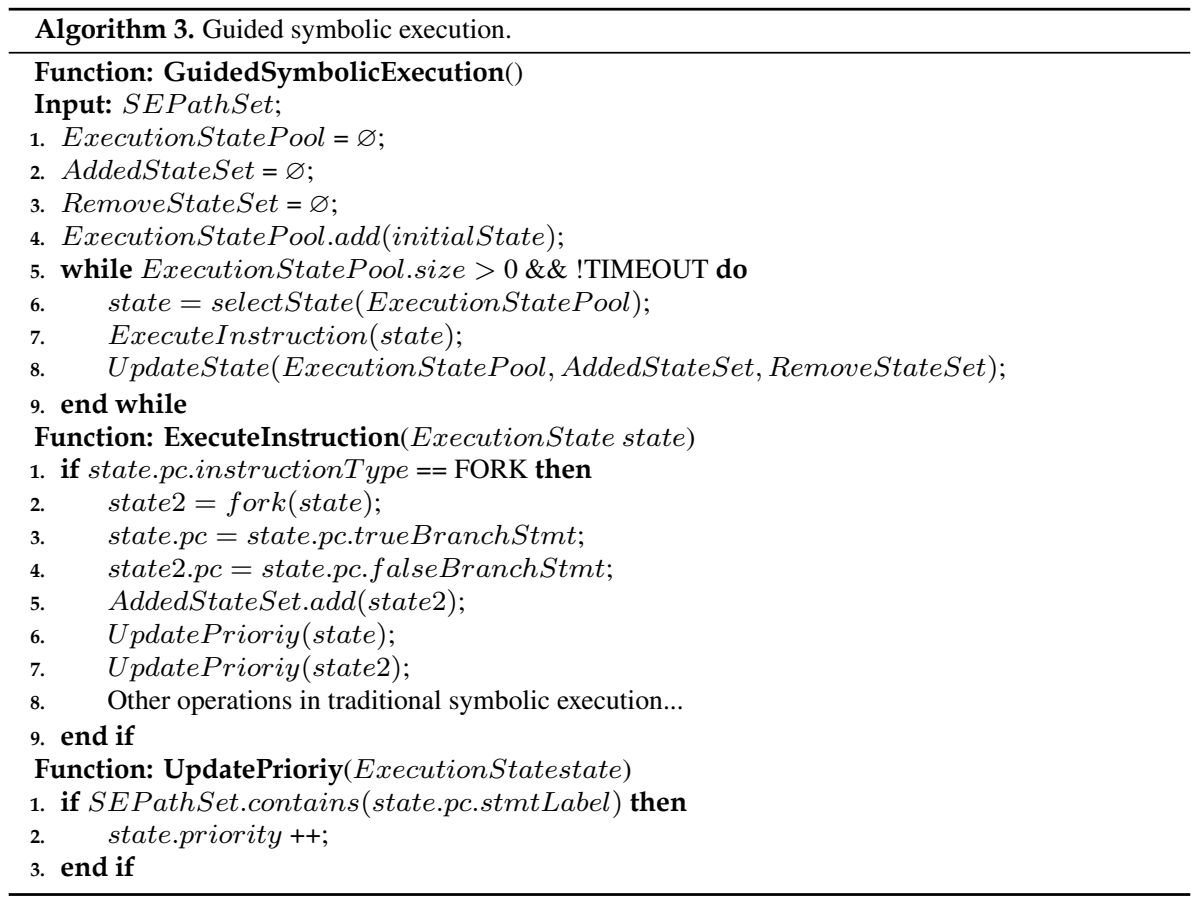

\subsection{Hybrid mechanism}

In general, model prediction is not $100 \%$ accurate. If symbolic execution (resp. fuzzing) cannot produce new branch coverage within designated time, for the purpose of tolerating path classification inaccuracy, SmartFuSE removes the uncovered paths from the guided path set, SEPPathSet (resp. FuzzPathSet), and transmits them to fuzzing (resp. symbolic execution) for another guiding to further improve coverage. If path prediction alone can show whether the paths are suitable for symbolic execution (resp. fuzzing) and subsequently, symbolic execution (resp. fuzzing) is guided, then such guiding strategy can rapidly increase the coverage of the paths of codes which are suitable for symbolic execution (resp. fuzzing). However, such guiding strategy does not apply to the paths that neither symbolic execution nor fuzzing can 
cover. Thus, we transmit all the test cases generated in the symbolic execution to fuzzing so that fuzzing can mutate to generate new inputs on the basis of these inputs to try to cover the paths that cannot be covered by both symbolic execution and fuzzing.

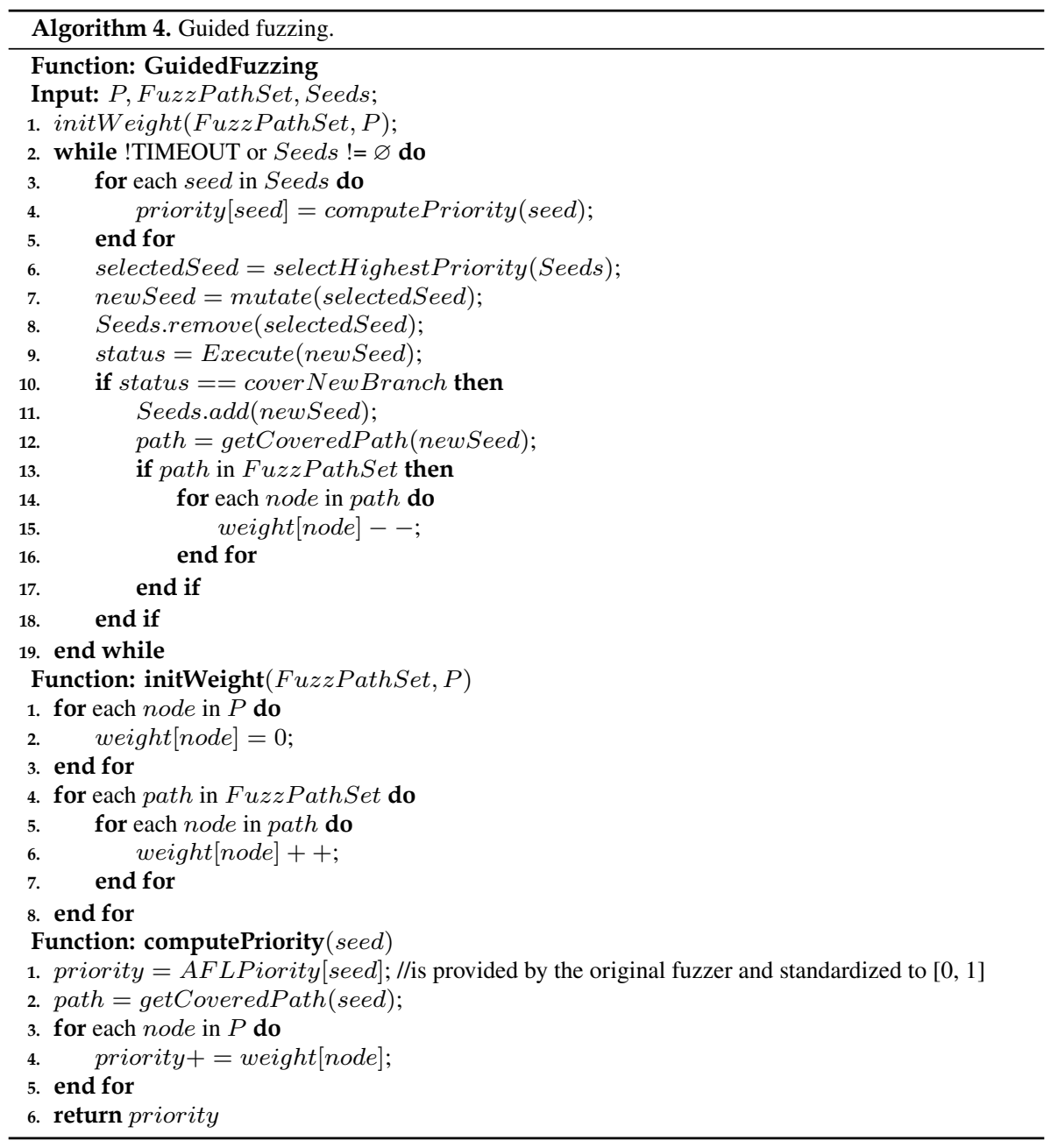

At the same time, the paths covered by fuzzing are switched to symbolic execution. When symbolic execution again explores these paths, we can choose to no longer undertake timeconsuming constraint solving. Instead, more time can be spent on exploring other paths.

\section{Implementation and Experiment}

\subsection{Implementation and experiment settings}

In this paper, we develop the hybrid testing tool SmartFuSE. Its overall framework is mainly based on Python. Tensorflow 1.4 is used to build the GGNN model. On the basis of KLEE and AFL, the model is modified into guided symbolic execution and fuzzing.

We select the common test objects of fuzzing, i.e., programs (Base64, MD5sum, Uniq, and Who) in LAVA-M ${ }^{[17]}$, which are inserted with many defects difficult to be detected. The 
effectiveness of SmartFuSE is tested on them. The answers to the following questions are tried to be found through experiments.

RQ1: What is the effect of guided symbolic execution and fuzzing for the paths predicted by the path classification prediction model of SmartFuSE?

RQ2: How are the optimization effects of path classification guiding and hybrid mechanism in SmartFuSE on fuzzing?

RQ3: What is the effect of defect detection of SmartFuSe compared to that of other hybrid testing and fuzzing tools?

The machine used to run the experiments is equipped with the CPU Intel i7-8700 K, a 32 GB memory, and Ubuntu 16.04 operation system. In order to train the model, the data is collected from the programs date, cat, dd, df, cp, and echo, part of Coreutils-6.11. The code coverage information of the programs is collected by the modified AFL-Cov ${ }^{[18]}$. For the purpose of display, when training data are collected, the running time of KLEE and AFL is set to 24 hours. In the LAVA-M project, the running time of SmartFuSE, KLEE, and AFL is also set to 24 hours. As the existing hybrid testing tools Afleer ${ }^{[2]}$ and PANGOLIN ${ }^{[19]}$ are not open-source, Driller ${ }^{[1]}$ cannot obtain certain dependences. Thus, we use the Python interface shellphuzz ${ }^{[20]}$ provided by Driller official code library to replace Driller.

\subsection{Experiment results}

To verify the accuracy of the path classification prediction model of SmartFuSE, we collect the paths of bzip2-1.0.6 that have been executed for 2 hours on KLEE and AFL and log the time of the test cases for covering the paths. Then, we compare the classification results with those of the path classification prediction model of SmartFuSE and find that the prediction accuracy of the SmartFuSE model reaches $84.7 \%$.

\subsubsection{Comparison of the code coverage effect of symbolic execution and fuzzing guided by the} paths predicted by the path classification prediction model of SmartFuSE on the LAVA-M programs (RQ1)

We make statistics of the statement coverage, branch coverage, and path number of KLEE and guided KLEE on the four programs of LAVA-M respectively (Table 1). In terms of statement coverage, the guided KLEE covers $1.5 \%$ more codes than KLEE. In terms of branch coverage, the guided KLEE covers $2.7 \%$ more branches than KLEE. In terms of path coverage, the guided KLEE increases by 5.5 folds compared with KLEE. In the table, only the number of paths of KLEE which the test cases generated by KLEE can cover is computed. As constraint solving is rather time-consuming, KLEE cannot generate a large number of test cases merely through mutation as AFL does. The paths suitable for KLEE predicted by the path classification prediction model of SmartFuSE are used to guide KLEE, which can effectively improve the code coverage of KLEE.

Table 1 Coverage information of KLEE and guided KLEE on LAVA-M

\begin{tabular}{|c|c|c|c|c|c|c|c|}
\hline \multirow{2}{*}{ Program } & \multirow{2}{*}{ LOC } & \multicolumn{2}{|c|}{ Statement coverage LCOV $(\%)$} & \multicolumn{2}{|c|}{ Branch coverage BCOV $(\%)$} & \multicolumn{2}{|c|}{ Path number } \\
\hline & & KLEE & Guided KLEE & KLEE & Guided KLEE & KLEE & Guided KLEE \\
\hline Base64 & 113 & 40.7 & 53.1 & 31.7 & 44.2 & 27 & 52 \\
\hline MD5sum & 416 & 28.8 & 37.3 & 20.8 & 25.4 & 1 & 3 \\
\hline Uniq & 260 & 77.7 & 84.2 & 64.5 & 69.2 & 66 & 62 \\
\hline Who & 4,073 & 97.4 & 97.4 & 27.2 & 29.2 & 31 & 691 \\
\hline Total & 4,862 & 89.2 & 90.7 & 33.8 & 36.5 & 125 & 808 \\
\hline
\end{tabular}

We make statistics of statement coverage, branch coverage, and path number of AFL and guided AFL on the four programs of LAVA-M respectively (Table 2). The statement coverage 
of guided AFL is not increased significantly as compared with AFL. In comparison with AFL, guided AFL achieves a 3\% increase in branch coverage and a 0.4 -fold increase in path coverage.

\subsubsection{Optimization effect of the guidance of path classification and hybrid mechanism in SmartFuSE on fuzzing (RQ2)}

We make statistics of statement coverage, branch coverage, and path number of KLEE, AFL, and SmartFuSE on the four programs of LAVA-M respectively (Table 3). In terms of statement coverage, SmartFuSE does not increase significantly compared with KLEE or AFL alone. In general, the code coverage of SmartFuSE is 3.4\% and 2.8\% higher than that of KLEE and AFL, respectively. In terms of branch coverage, SmartFuSE is $26.9 \%$ and $20.7 \%$ higher than KLEE and AFL, respectively. Regarding path coverage, SmartFuSE can notably cover more paths than KLEE and AFL, achieving 13.5 and 0.9 times of increase compared with KLEE and AFL, respectively.

Table 2 Coverage information of AFL and guided AFL on LAVA-M

\begin{tabular}{|c|c|c|c|c|c|c|c|}
\hline \multirow{2}{*}{ Program } & \multirow{2}{*}{ LOC } & \multicolumn{2}{|c|}{ Statement coverage LCOV(\%) } & \multicolumn{2}{|c|}{ Branch coverage $\mathrm{BCOV}(\%)$} & \multicolumn{2}{|c|}{ Path number } \\
\hline & & AFL & Guided AFL & AFL & Guided AFL & AFL & Guided AFL \\
\hline Base64 & 113 & 39.8 & 40.7 & 33.7 & 34.6 & 339 & 439 \\
\hline MD5sum & 416 & 68.5 & 68.5 & 51.7 & 51.7 & 309 & 368 \\
\hline Uniq & 260 & 47.3 & 47.3 & 26.5 & 26.5 & 106 & 150 \\
\hline Who & 4,073 & 96 & 96.1 & 29.3 & 30.2 & 181 & 377 \\
\hline Total & 4,862 & 89.77 & 89.82 & 40.0 & 40.3 & 935 & 1334 \\
\hline
\end{tabular}

Table 3 Coverage information on LAVA-M

\begin{tabular}{|c|c|c|c|c|c|c|c|c|c|c|}
\hline \multirow{2}{*}{ Program } & \multirow{2}{*}{ LOC } & \multicolumn{3}{|c|}{ Statement coverage LCOV $(\%)$} & \multicolumn{3}{|c|}{ Branch coverage BCOV $(\%)$} & \multicolumn{3}{|c|}{ Path number } \\
\hline & & KLEE & AFL & SmartFuSE & KLEE & AFL & SmartFuSE & KLEE & AFL & SmartFuSE \\
\hline Base64 & 113 & 40.7 & 39.8 & 40.7 & 31.7 & 33.7 & 35.6 & 27 & 339 & 413 \\
\hline MD5sum & 416 & 28.8 & 68.5 & 69.0 & 20.8 & 51.7 & 30.1 & 1 & 309 & 285 \\
\hline Uniq & 260 & 77.7 & 47.3 & 77.7 & 64.5 & 26.5 & 64.5 & 66 & 106 & 151 \\
\hline Who & 4,073 & 97.4 & 96 & 97.4 & 27.2 & 29.3 & 61.8 & 31 & 181 & 963 \\
\hline Total & 4,862 & 89.2 & 89.8 & 92.6 & 33.8 & 40.0 & 60.7 & 125 & 935 & 1812 \\
\hline
\end{tabular}

Next, we will further analyze the defect detection abilities of SmartFuSe. Apart from existing defects, each program of LAVA-M is artificially inserted with some defects (44 in Base64, 57 in MD5sum, 28 in Uniq, and 2,136 in Who), with results shown in Figure 3.

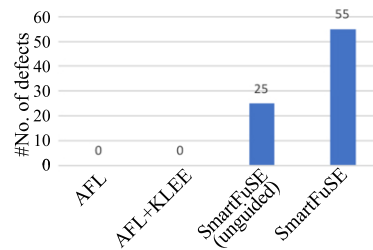

(a) Base64

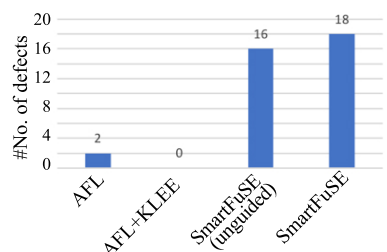

(b) Uniq

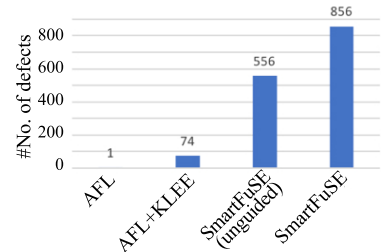

(c) Who

Figure 3 Number of defects detected by SmartFuSE, AFL, and KLEE on LAVA-M

By analyzing the experiment results, we find that SmartFuSE is much better than KLEE and AFL in detecting defects in the programs in LAVA-M. After KLEE runs independently for 24 hours, it cannot detect any defect in any of the LAVA-M programs. In comparison, after AFL runs for 24 hours independently, it can detect one to two defects. The phenomena can be explained as follows. KLEE, despite its satisfactory statement coverage, covers only a small number of paths. AFL depends on good test input. It is only provided with the test input given in LAVA-M. Because the number of seeds is small, AFL cannot generate multiple test 
cases through mutation. During the $24 \mathrm{~h}$ simultaneous operation of AFL and KLEE, the test cases of KLEE are transmitted to AFL. Through such simple AFL + KLEE, 74 defects can be detected in the who program. It indicates that KLEE can cover the code space differently from that of AFL and transmitting the test cases from KLEE to AFL can help AFL explore new code space. However, the defect detection capability of AFL + KLEE remains to be improved. On the basis of AFL + KLEE, SmartFuSE (unguided) introduces a hybrid mechanism to make AFL first mutate the seeds with new branch coverage transmitted from KLEE so that KLEE no longer performs constraint solving for the paths covered by AFL to avoid repeatedly generating test cases. Figure 3 shows that SmartFuSE (unguided) can detect more than 500 defects more than AFL + KLEE. This proves that the hybrid mechanism proposed in this paper can improve the execution effect of AFL and KLEE. Furthermore, on the basis of SmartFuSE (unguided), SmartFuSE increases the number of paths predicted by the path classification prediction model, guides symbolic execution and fuzzing, and copes with prediction errors. Figure 3 also presents that after SmartFuSE runs for 24 hours, it detects not only 44 defects artificially inserted in Base64 but also another 11 defects in it, 18 defects in the Uniq program, and 856 defects in the Who program, totaling 929 bugs. In the MD5sum program, SmartFuSE does not detect any defect. Through investigation, we find that it is caused by the hash operation in the program ${ }^{[2]}$. In summary, Figure 3 indicates that the path classification guiding and the hybrid mechanism proposed in this paper can help KLEE and AFL detect more program defects.

\subsubsection{Comparison of the effect of defect detection between SmartFuSe and other hybrid testing and fuzzing tools (RQ3)}

We discover some execution errors in the utilization of Driller (shellphuzz), which may affect the test results. Thus, we refer to the experimental results in PANGOLIN ${ }^{[9]}$, and compare the effects between SmartFuSE and some existing hybrid testing and fuzzing tools. The experimental results of AFL are achieved by two AFL processes, while the experimental results of AFL mentioned above are obtained from one AFL process. Figure 4 shows the number of defects detected by hybrid fuzzing tools (PANGOLIN, QSYM ${ }^{[5]}$, Driller, and SmartFuSE) and fuzzing tools (AFL, AFLFast ${ }^{[21]}$, Angora ${ }^{[22]}$, and T-Fuzz ${ }^{[23]}$ ) in the LAVA-M program set in 24 hours respectively. SmartFuSE discovers the most defects in the base64 and shows the detection capacities at a moderate level for Uniq and who (Figure 4). Among four projects, SmartFuSE can detect more defects than AFL and Driller. We will discuss the features of these tools in the section of related work in this paper.

\subsection{Experimental discussion}

The analysis of experimental results demonstrates that SmartFuSE is much better than KLEE and AFL in detecting defects in the LAVA-M program set. We further analyze the test cases that can trigger defects in SmartFuSE fuzzing. We find that many test cases are the new test cases obtained by constant mutation on the basis of the test cases generated in the symbolic execution module in SmartFuSE. Moreover, through guided symbolic execution, SmartFuSE can more quickly explore paths suitable for symbolic execution and generate more test cases. The symbolic execution phase provides more test cases for the fuzzing phase and helps fuzzing explore paths that cannot be easily covered by fuzzing to generate test cases with higher code coverage, so that more program defects can be detected. It proves that the hybrid mechanism incorporating symbolic execution and fuzzing does help SmartFuSE more quickly cover the paths that cannot be easily covered by both symbolic execution and fuzzing, which makes the defect detection ability of SmartFuSE better and more effective than symbolic execution or fuzzing alone. 

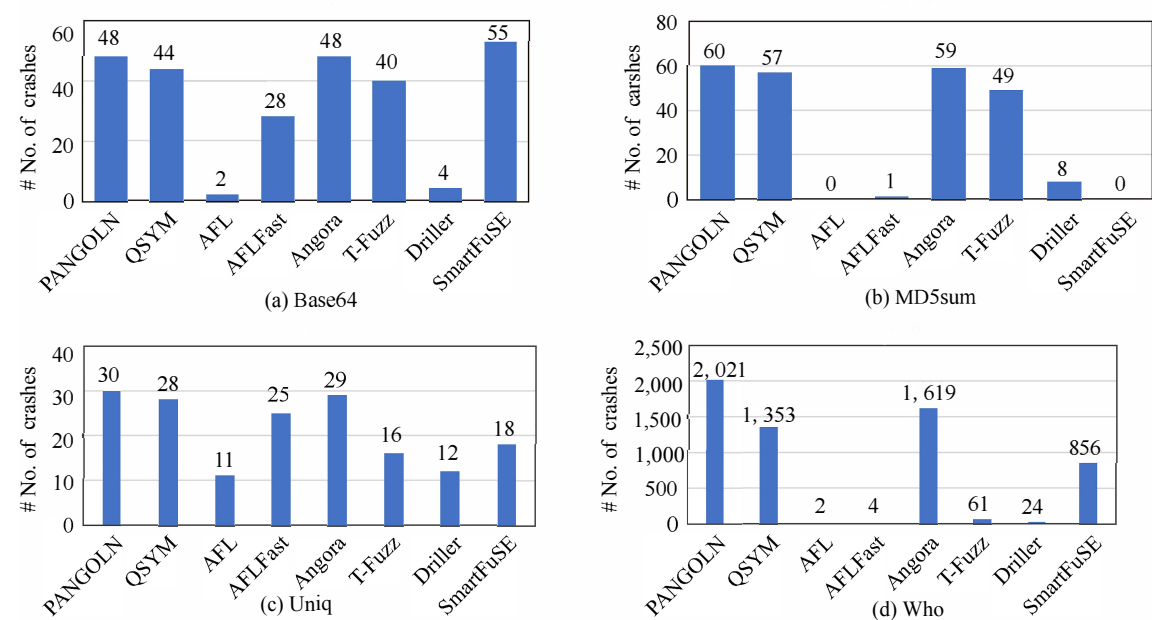

Figure 4 Number of defects detected by several fuzzing and hybrid tools on LAVA-M

The following factors may affect the effectiveness of tools. (1) The data set richness can affect the accuracy of the path classification prediction model of SmartFuSE and the utility of the tool. Plenty of data sets are required to train neural networks. However, there are no relevant data sets available. To lower the requirement for the number of data sets in the experiment, we select small- and medium-scale programs to generate data sets. As these programs have been extensively used by KLEE, KLEE can generate test cases easily. In contrast, too much time and memory is needed by KLEE to execute programs of large scale and solve constraints to generate test cases. Hence, it is impossible to collect enough data sets within a short time. This is the problem that we should solve in our follow-up research. Furthermore, the prediction model may have higher prediction accuracy for the programs of training sets, but it does not have satisfactory prediction accuracy for other programs. (2) The accuracy of the path classification prediction model of SmartFuSE can affect the execution efficiency of SmartFuSE hybrid testing. Model prediction accuracy, in general, cannot reach $100 \%$. Inaccurate path classification prediction will cause SmartFuSE to guide symbolic execution (resp. fuzzing) to explore paths unsuitable for it. To reduce the negative impact caused by inaccurate path classification prediction, we set a guided time threshold. If within a certain time symbolic execution (resp. fuzzing) cannot be guided to cover the path, SmartFuSE shifts to fuzzing (resp. symbolic execution) for the path coverage. (3) The time threshold setting can affect the effect of execution. If the time threshold is too large, inaccurate prediction will be attempted too long. If the time threshold is too small, accurate prediction (e.g., suitable for symbolic execution) will be attempted by an unsuitable method (e.g., fuzzing), which makes the corresponding paths unable to be covered.

\section{Related Work}

In this section, we will outline relevant studies from the aspects of symbolic execution, fuzzing, and the hybrid testing incorporating the two.

\subsection{Symbolic execution}

Symbolic execution is a common technique for software analysis. Conventional symbolic execution faces the problems of path explosion, environment interaction, system calls, and constraint solving ${ }^{[24]}$. To address such problems, a number of optimization techniques have been put forward. 
To cope with the environment interaction and system calls, $\mathrm{KLEE}^{[9]}$ and $\mathrm{AEG}^{[25]}$ established abstract models for system calls to support symbolic files and sockets as the inputs of the symbolic execution. In selective symbolic execution, for example, the S2E platform ${ }^{[26]}$, based on KLEE and the QEMU virtual machine, supports target code in undertaking symbolic execution in the program through simultaneously performing single-path execution of concrete values and multi-path execution of symbolic values. Concrete execution is carried out when the kernel function of the system is called so as to realize the smooth switch between symbolic execution and concrete execution.

To tackle the path explosion, many studies come up with different solutions. Some propose optimization algorithms for path selection ${ }^{[9,25,27-30]}$, i.e., searching strategies for symbolic execution. For example, KLEE provides many strategies for path searching, including Depth First Search (DFS), Breadth First Search (BFS), random state search, and random path search. Some propose function summary ${ }^{[31-33]}$ and loop summary ${ }^{[34,35]}$. Through the multiplexing of the execution information of explored code, they can be prevented from being executed again, which thus improves the efficiency of symbolic execution. Some studies effectively reduce the traversal paths through state merging ${ }^{[36,37]}$, i.e., merging several paths into one state. However, state merging makes path constraints even more complex, increasing the difficulty of constraint solving. Some studies trim uninteresting paths by working with other program analysis techniques ${ }^{[38-42]}$, such as program slicing, taint analysis, type checking, and compiling optimization, so that symbolic execution can focus on analyzing code space that can more easily discover errors and defects.

To tackle the problem of constraint solving, $\mathrm{Z}^{[43]}$, which is the current mainstream SMT constraint solver, is extensively adopted by symbolic execution engines, such as KLEE, Mayhem ${ }^{[39]}$, and Angr ${ }^{[44]}$. EXE ${ }^{[4]}$, KLEE, and AEG also use the $\mathrm{STP}^{[46]}$ constraint solver. In addition, some symbolic execution engines are optimized to address the problem of constraint solving. For example, EXE, KLEE, and S2E simplify the collected constraints to reduce the constraint complexity. Symbolic execution engines EXE, KLEE, Memoise ${ }^{[47]}$, and GreenTrie ${ }^{[48]}$ cache the constraint solutions to reduce the calling of solvers.

The proposed method, integrating symbolic execution and fuzzing, is conducive to solving the problems of environment interaction and constraint solving.

\subsection{Fuzzing}

Fuzzing techniques can be divided into three categories, i.e., black-box fuzzing, whitebox fuzzing, and gray-box fuzzing ${ }^{[49]}$. Black-box fuzzing does not analyze the program itself. Instead, in line with the preset rules, it mutates and generates new seeds. For example, fuzzing based on genetic algorithms mutates and generates new inputs through bit flipping, byte copying, and byte deletion. Fuzzing based on syntax automatically generates new seeds following syntax rules $^{[50]}$. In contrast, white-box fuzzing analyzes the information of the program itself, including control flow and data flow. White-box fuzzing was first proposed by Godefroid et al.$^{[51,52]}$. The tool SAGE implementing this method collects path constraints from the actual execution of concrete inputs, and according to the principle of code coverage maximization, solves the constraint system after address inversion to generate new test inputs to proceed with fuzzing. Gray-box fuzzing is a compromise between black-box fuzzing and white-box fuzzing. It is often applied to code instrumentation ${ }^{[53,54]}$ and taint analysis ${ }^{[55]}$.

To improve the code coverage and the execution efficiency of fuzzing, many studies propose improving methods. BuzzFuzz ${ }^{[56]}$ used dynamic taint analysis to identify parts of inputs correlated with target defects and mutated them to generate new test inputs. AFLFast ${ }^{[21]}$ gave higher priority and longer mutation time to the test inputs that can cover low-frequency 
paths by estimating seeds with a Markov chain model. To make fuzzing more quickly cover the given target positions, AFLg ${ }^{[57]}$ proposed guided fuzzing. The distance from seeds to the targets was computed, and then the Markov Chain Monte Carlo (MCMC) technique was used to select seeds closer to targets; such seeds were given higher priorities and longer mutation time. VUzzer ${ }^{[58]}$ identified magic bytes of inputs through dynamic taint analysis and then mutated magic bytes to generate new inputs. Steelix ${ }^{[59]}$ used lightweight static analysis and binary instrumentation to position magic bytes of inputs. $\mathrm{NEUZZ}^{[60]}$ used a neural network model to learn the smoothed approximation of the branch behaviors of the program and mutated test cases with a gradient guiding strategy so as to smooth the search process of fuzzing. Skyfire ${ }^{[61]}$ proposed a data-driven method to generate seeds. With a large number of existing test inputs, this method learned the syntax and semantic information of inputs. On this basis, inputs could be generated for the fuzzing of the tested program with highly structured inputs. To solve path constraints without resorting to symbolic execution, Angora ${ }^{[22]}$ proposed the byte-level taint analysis and rapidly found the solution that satisfied the constraints of complex paths through the gradient descent method so as to obtain new inputs for fuzzing. T-Fuzz ${ }^{[23]}$ put forward fuzzing tools for communication protocols by modelling the TTCN-3 model.

\subsection{Hybrid testing incorporating symbolic execution and fuzzing}

For binary code, Driller ${ }^{[1]}$ incorporated a hybrid execution engine Angr into AFL. When fuzzing met some special boundary conditions (such as narrow paths that satisfy specific inputs), a binary hybrid testing module (Angr) would be used. Through constraint solution, this module can generate new inputs that can overcome these limitations and feed inputs to fuzzing as seeds so that fuzzing can proceed. DigFuzz ${ }^{[3]}$ proposed to use a Monte Carlo probability model to calculate the priorities of paths executed in fuzzing and solve more difficult paths through hybrid testing. In the hybrid fuzzing proposed by Pak et al. ${ }^{[4]}$, symbolic execution was first used to explore the space of the programs. Then, the obtained bytes of key parts of paths corresponding to test inputs remained unchanged while bytes of other parts were randomly mutated to generate new inputs before they were explored by fuzzing. Similarly, QSYM ${ }^{[5]}$ solved the constraints of some paths in the hybrid testing to generate test cases serving as basic seeds. Then, on the basis of these seeds, test inputs meeting the requirement were generated through mutation. SYMFUZZ $^{[6]}$ first obtained the dependences between input bits by analyzing the symbolic execution tracks of two input seeds in the black-box fuzzing. Then the best mutation rate of seeds was computed on the basis of such dependences. The newly generated seeds were taken as the new inputs of fuzzing. Munch ${ }^{[7]}$ proposed a coarse-grained hybrid fuzzing method. To improve function coverage, Munch first used fuzzing to run the program and then used guided symbolic execution to explore the functions not covered in fuzzing. The generated new test inputs were also subjected to fuzzing. Afleer ${ }^{[2]}$ proposed a fine-grained hybrid fuzzing method, in which source code instrumentation was performed to generate LLVM intermediate code and executable files after instrumentation, and then simultaneously ran fuzzing and symbolic execution. Fuzzing continuously generated new test cases and updated coverage information. Symbolic execution systematically explored the state space of the program and generated test cases for uncovered branches as seeds of fuzzing. In this method, symbolic execution would inevitably explore paths unsuitable for such a tool. In comparison, SmartFuSE performs the classification prediction of paths and accordingly guides the symbolic execution and fuzzing so that they would first explore paths suitable for them. In this way, symbolic execution allocates more time to explore and solve paths suitable for it in the early phase. Meanwhile, fuzzing is guided to first explore paths that are predicted to be unsuitable for symbolic execution and those discovered unsuitable after some time of execution. As a result, the path coverage of SmartFuSE can be further improved. PANGOLIN ${ }^{[19]}$ proposed an incremental hybrid fuzzing. 
Uncovered paths were abstracted by polyhedrons to guide seed mutation and hybrid testing to generate numerous test inputs within polynomial time. SeededFuzz ${ }^{[62]}$ used static analysis, dynamic monitoring, and symbolic execution to generate and select suitable seeds. With respect to the Sequence Directed Hybrid Fuzzing (SDHF) proposed by Berry ${ }^{[63]}$, when the statement sequence of a program was given, this method enhanced the ability of fuzzing by sequence directing strategy and hybrid execution. ILF $^{[64]}$ proposed an imitation learning based fuzzer. In this method, a neural network model learned the high-quality test cases generated from the coverage-based symbolic execution so as to acquire the strategy of generating seeds in fuzzing. The method improved the seed quality in fuzzing with symbolic execution as learning objects, while the method proposed in this paper completes the hybrid testing by allocating suitable paths to symbolic execution and fuzzing respectively through deep learning. At present, none of the existing hybrid testing methods incorporating symbolic execution and fuzzing applied deep learning to allocate paths suitable for either symbolic execution or fuzzing and none of them, on this basis, guided symbolic execution and fuzzing to conduct hybrid testing.

\section{Conclusion}

In this paper, we proposed a deep learning-based hybrid testing method incorporating symbolic execution and fuzzing. With symbolic executor KLEE and fuzzer AFL, we implemented SmartFuSE integrating symbolic execution and fuzzing. We tested several programs in the LAVA-M and found that our tool SmartFuSE, compared with independent fuzzing or symbolic execution, can not only improve code coverage but also significantly improve the ability to detect defects.

Although this work reveals with deep learning that symbolic execution and fuzzing have their own different preference for the exploration of code space, the respective features of code space suitable for symbolic execution and fuzzing are not provided. In the future, we expect to figure out these features, which can provide references for symbolic execution, fuzzing, and the hybrid testing integrating the two.

\section{References}

[1] Stephens N, Grosen J, Salls C, et al. Driller: Augmenting fuzzing through selective symbolic execution. Proc. of the Network and Distributed System Security Symp. (NDSS). 2016.

[2] Xie X, Li X, Chen X, et al. Branch coverage-guided hybrid testing based on symbolic execution and fuzzing. Ruan Jian Xue Bao/Journal of Software, 2019, 30(10): 3071-3089. http://www.jos.org.cn/ 1000-9825/5789.htm. [doi: 10.13328/j.cnki.jos.005789]

[3] Zhao L, Duan Y, Yin H, et al. Send hardest problems my way: Probabilistic path prioritization for hybrid fuzzing. Proc. of the Network and Distributed System Security Symp. (NDSS). 2019.

[4] Pak BS. Hybrid fuzz testing: Discovering software bugs via fuzzing and symbolic execution [Ph.D. Thesis]. Pittsburgh: School of Computer Science, Carnegie Mellon University, 2012.

[5] Yun I, Lee S, Xu M, et al. QSYM: A practical concolic execution engine tailored for hybrid fuzzing. Proc. of the 27th USENIX Security Symp. (USENIX Security 18). 2018. 745-761.

[6] Cha SK, Woo M, Brumley D. Program-adaptive mutational fuzzing. Proc. of the IEEE Symp. on Security and Privacy (SP). IEEE, 2015. 725-741.

[7] Ognawala S, Hutzelmann T, Psallida E, et al. Improving function coverage with munch: A hybrid fuzzing and directed symbolic execution approach. Proc. of the 33rd Annual ACM Symp. on Applied Computing. 2018. 1475-1482.

[8] King JC. Symbolic execution and program testing. Communications of the ACM, 1976, 19(7): 385394.

[9] Cadar C, Dunbar D, Engler DR. KLEE: Unassisted and automatic generation of high-coverage tests 
for complex systems programs. Proc. of the USENIX Symp. on Operating Systems Design and Implementations (OSDI). 2008. 209-224.

[10] Miller BP, Fredriksen L, So B. An empirical study of the reliability of UNIX utilities. Communications of the ACM, 1990, 33(12): 32-44.

[11] McNally R, Yiu K, Grove D, et al. Fuzzing: The state of the art. Defence Science and Technology Organisation Edinburgh (Australia), 2012. http://citeseerx.ist.psu.edu/viewdoc/download;jsessionid= 15CF9A7FD272D62D76FB5ED26DA3808F?doi=10.1.1.461.4627\&rep=rep1\&type=pdf.

[12] American fuzzy lop. 2020. http://lcamtuf.coredump.cx/afl/.

[13] Si XJ, Dai HJ, Raghothaman M, et al. Learning loop invariants for program verification. Advances in Neural Information Processing Systems (NeurIPS). 2018. 7751-7762.

[14] Li YJ, Tarlow D, Brockschmidt M, et al. Gated graph sequence neural networks. arXiv: 1511.05493. 2015.

[15] Cho K, Van Merriënboer B, Bahdanau D, et al. On the properties of neural machine translation: Encoder-decoder approaches. arXiv: 1409.1259. 2014.

[16] Ferrante J, Ottenstein KJ, Warren JD. The program dependence graph and its use in optimization. ACM Trans. on Programming Languages and Systems (TOPLAS), 1987, 319-349.

[17] Dolan-Gavitt B, Hulin P, Kirda E, et al. Lava: Large-scale automated vulnerability addition. Proc. of the IEEE Symp. on Security and Privacy (SP). IEEE 2016. 110-121.

[18] AFL Cov. 2020. http://cipherdyne.com/afl-cov/.

[19] Huang H, Yao P, Wu R, et al. PANGOLIN: Incremental hybrid fuzzing with polyhedral path abstraction. Proc. of the IEEE Symp. on Security and Privacy (SP). 2020. 1613-1627.

[20] Shellphuzz. 2020. https://github.com/shellphish/fuzzer.

[21] Böhme M, Pham VT, Roychoudhury A. Coverage-based greybox fuzzing as markov chain. IEEE Trans. on Software Engineering, 2017, 45(5): 489-506.

[22] Chen P, Chen H. Angora: Efficient fuzzing by principled search. Proc. of the IEEE Symp. on Security and Privacy (SP). IEEE, 2018. 711-725.

[23] Johansson W, Svensson M, Larson UE, et al. T-Fuzz: Model-based fuzzing for robustness testing of telecommunication protocols. Proc. of the 17th IEEE Int'1 Conf. on Software Testing, Verification and Validation. IEEE, 2014. 323-332.

[24] Baldoni R, Coppa E, D'elia DC, et al. A survey of symbolic execution techniques. ACM Computing Surveys (CSUR), 2018, 51(3): 1-39.

[25] Avgerinos T, Cha SK, Rebert A, et al. Automatic exploit generation. Communications of the ACM, 2014, 57(2): 74-84.

[26] Chipounov V, Kuznetsov V, Candea G. The S2E platform: Design, implementation, and applications. ACM Trans. on Computer Systems (TOCS), 2012, 30(1): 1-49.

[27] Li Y, Su Z, Wang L, et al. Steering symbolic execution to less traveled paths. ACM SIGPLAN Notices, 2013, 48(10): 19-32.

[28] Ma KK, Phang KY, Foster JS, et al. Directed symbolic execution. Proc. of the Int'1 Static Analysis Symp. Springer-Verlag, 2011. 95-111.

[29] Zhang Y, Chen Z, Wang J, et al. Regular property guided dynamic symbolic execution. Proc. of the 37th IEEE/ACM Int'1 Conf. on Software Engineering (ICSE). IEEE, 2015. 643-653.

[30] Xie T, Tillmann N, De Halleux J, et al. Fitness-guided path exploration in dynamic symbolic execution. Proc. of the IEEE/IFIP Int'l Conf. on Dependable Systems \& Networks (DSN). IEEE, 2009. 359-368.

[31] Godefroid P. Compositional dynamic test generation. Proc. of the 34th Annual ACM SIGPLANSIGACT Symp. on Principles of Programming Languages (POPL). 2007. 47-54.

[32] Anand S, Godefroid P, Tillmann N. Demand-driven compositional symbolic execution. Proc. of the Int'l Conf. on Tools and Algorithms for the Construction and Analysis of Systems. Springer-Verlag, 2008. 367-381.

[33] Boonstoppel P, Cadar C, Engler D. RW set: Attacking path explosion in constraint-based test generation. 
Proc. of the Int'1 Conf. on Tools and Algorithms for the Construction and Analysis of Systems. SpringerVerlag, 2008. 351-366.

[34] Godefroid P, Luchaup D. Automatic partial loop summarization in dynamic test generation. Proc. of the Int'l Symp. on Software Testing and Analysis (ISSTA). 2011. 23-33.

[35] Xie X, Chen B, Liu Y, et al. Proteus: Computing disjunctive loop summary via path dependency analysis. Proc. of the 24th ACM SIGSOFT Int'l Symp. on Foundations of Software Engineering (FSE). 2016. 61-72.

[36] Hansen T, Schachte P, Søndergaard H. State joining and splitting for the symbolic execution of binaries. Proc. of the Int'1 Workshop on Runtime Verification. Springer-Verlag, 2009. 76-92.

[37] Kuznetsov V, Kinder J, Bucur S, et al. Efficient state merging in symbolic execution. ACM SIGPLAN Notices, 2012, 47(6): 193-204.

[38] Shoshitaishvili Y, Wang R, Hauser C, et al. Firmalice-automatic detection of authentication bypass vulnerabilities in binary firmware. Proc. of the Network and Distributed System Security Symp. (NDSS). 2015.

[39] Cha SK, Avgerinos T, Rebert A, et al. Unleashing mayhem on binary code. Proc. of the IEEE Symp. on Security and Privacy. IEEE, 2012. 380-394.

[40] Khoo YP, Chang BY, Foster JS. Mixing type checking and symbolic execution. ACM SIGPLAN Notices, 2010, 45(6): 436-47.

[41] Gao F, Wang L, Li X. BovInspector: Automatic inspection and repair of buffer overflow vulnerabilities. Proc. of the 31st IEEE/ACM Int'l Conf. on Automated Software Engineering (ASE). IEEE, 2016. 786791.

[42] Wang G, Chattopadhyay S, Biswas AK, et al. Kleespectre: Detecting information leakage through speculative cache attacks via symbolic execution. ACM Trans. on Software Engineering and Methodology (TOSEM), 2020, 29(3): 1-31.

[43] De Moura L, Bjørner N. Z3: An efficient SMT solver. Proc. of the Int'1 Conf. on Tools and Algorithms for the Construction and Analysis of Systems. Springer-Verlag, 2008. 337-340.

[44] Shoshitaishvili Y, Wang R, Salls C, et al. SoK: (state of) the art of war: Offensive techniques in binary analysis. Proc. of the IEEE Symp. on Security and Privacy (SP). IEEE, 2016. 138-157.

[45] Cadar C, Ganesh V, Pawlowski PM, et al. EXE: Automatically generating inputs of death. Proc. of the 13th ACM Conf. on Computer and Communications Security (CCS). 2006.

[46] Ganesh V, Dill DL. A decision procedure for bit-vectors and arrays. Proc. of the Int'1 Conf. on Computer Aided Verification. Springer-Verlag, 2007. 519-531.

[47] Yang G, Păsăreanu CS, Khurshid S. Memoized symbolic execution. Proc. of the Int'l Symp. on Software Testing and Analysis (ISSTA). 2012. 144-154.

[48] Jia X, Ghezzi C, Ying S. Enhancing reuse of constraint solutions to improve symbolic execution. Proc. of the Int'l Symp. on Software Testing and Analysis (ISSTA). 2015. 177-187.

[49] Liang H, Pei X, Jia X, et al. Fuzzing: State of the art. IEEE Trans. on Reliability, 2018, 67(3): $1199-218$.

[50] Kim SY, Cha S, Bae DH. Automatic and lightweight grammar generation for fuzz testing. Computers \& Security, 2013, 1(36): 1.

[51] Godefroid P, Levin MY, Molnar DA. Automated whitebox fuzz testing. Proc. of the Network and Distributed System Security Symp. (NDSS). 2008. 151-166.

[52] Godefroid P, Levin MY, Molnar D. SAGE: Whitebox fuzzing for security testing. Queue, 2012, 10(1): 20-27.

[53] Luk CK, Cohn R, Muth R, et al. Pin: Building customized program analysis tools with dynamic instrumentation. ACM SIGPLAN Notices, 2005, 40(6): 190-200.

[54] Nethercote N, Seward J. Valgrind: A framework for heavyweight dynamic binary instrumentation. ACM SIGPLAN Notices, 2007, 42(6): 89-100.

[55] Bekrar S, Bekrar C, Groz R, et al. Finding software vulnerabilities by smart fuzzing. Proc. of the 4th IEEE Int'1 Conf. on Software Testing, Verification and Validation. IEEE, 2011. 427-430. 
[56] Fayaz SK, Yu T, Tobioka Y, et al. BUZZ: Testing context-dependent policies in stateful networks. In: Proc. of the 13th USENIX Symp. on Networked Systems Design and Implementation (NSDI 16). 2016. 275-289.

[57] Böhme M, Pham VT, Nguyen MD, et al. Directed greybox fuzzing. Proc. of the ACM SIGSAC Conf. on Computer and Communications Security (CCS). 2017. 2329-2344.

[58] Rawat S, Jain V, Kumar A, et al. VUzzer: Application-aware evolutionary fuzzing. Proc. of the Network and Distributed System Security Symp. (NDSS). 2017. 1-14.

[59] Li Y, Chen B, Chandramohan M, et al. Steelix: Program-state based binary fuzzing. Proc. of the 11th Joint Meeting on Foundations of Software Engineering. 2017. 627-637.

[60] She D, Pei K, Epstein D, et al. NEUZZ: Efficient fuzzing with neural program smoothing. Proc. of the IEEE Symp. on Security and Privacy (SP). IEEE, 2019. 803-817.

[61] Wang J, Chen B, Wei L, et al. Skyfire: Data-driven seed generation for fuzzing. Proc. of the IEEE Symp. on Security and Privacy (SP). IEEE, 2017. 579-594.

[62] Wang W, Sun H, Zeng Q. SeededFuzz: Selecting and generating seeds for directed fuzzing. Proc. of the Int'1 Symp. on Theoretical Aspects of Software Engineering. IEEE, 2016.

[63] Liang H, Jiang L, Ai L, et al. Sequence directed hybrid fuzzing. Proc. of the 27th IEEE Int'l Conf. on Software Analysis, Evolution and Reengineering (SANER). IEEE, 2020. 127-137.

[64] He J, Balunović M, Ambroladze N, et al. Learning to fuzz from symbolic execution with application to smart contracts. Proc. of the ACM SIGSAC Conf. on Computer and Communications Security (CCS). 2019. 531-548.

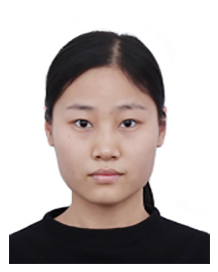

Fengjuan Gao bachelor. Her research interests include the software engineering, software analysis, software test, and software security.

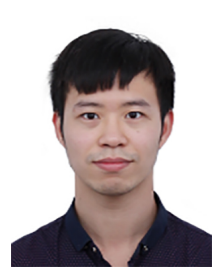

Yu Wang bachelor. His research interests include the software engineering, software analysis, software test, and software security.

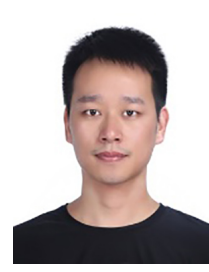

Lingyun Situ Ph.D., professional member of CCF. His research interests include the software engineering, information security, statistic analysis, and fuzzing.

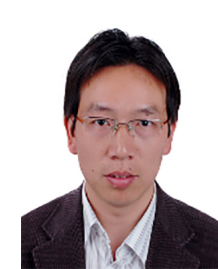

Linzhang Wang Ph.D., professor, doctoral supervisor, distinguished member of CCF. His research interests include the software engineering, software test, and software security. 